\title{
Análise de comentários espontâneos elaborados por usuários de ecstasy em pesquisa on-line
}

\author{
Stella Pereira Almeida, ${ }^{1}$ Juliana Bizeto ${ }^{1}$ e Maria Teresa Araujo Silva ${ }^{1}$
}

Como citar Almeida SP, Bizeto J, Silva MTA. Análise de comentários espontâneos elaborados por usuários de ecstasy em pesquisa on-line. Rev Panam Salud Publica. 2007;22(6):389-95.

RESUMO Objetivo. Analisar o conteúdo dos comentários enviados espontaneamente por usuários de ecstasy que responderam a um questionário on-line correspondente à primeira etapa de um projeto de redução de danos (projeto Baladaboa) para usuários dessa droga.

Método. Dos 1140 indivíduos que responderam o questionário, 412 enviaram comentários. Os comentários foram segmentados em cinco categorias (irrelevante, drogas, depoimentos, questionário e projeto) e submetidos à análise de conteúdo. A categoria "irrelevante" incluiu saudações, comentários irreverentes e manifestações ideológicas. A categoria "drogas" incluiu considerações sobre as causas, os efeitos e as conseqüências do uso de ecstasy ou de outras drogas, solicitações de esclarecimentos sobre a droga ou comentários que apontavam a informação como uma questão fundamental relacionada a seu uso. A categoria "depoimentos" incluiu comentários acerca de vivências pessoais. A categoria "questionário" incluiu comentários sobre o questionário propriamente dito, a formulação das questões, o uso de certos critérios e a solicitação de acesso aos resultados da pesquisa. A categoria "projeto" incluiu comentários relativos ao projeto Baladaboa.

Resultados. Identificaram-se 915 segmentos nos 412 comentários. A porcentagem de segmentos classificados em cada categoria foi: irrelevante, 9,8\%; drogas, 16,4\%; depoimentos, 18,1\%; questionário, 25,1\%; e projeto, 30,6\%. A maior parte dos comentários foi positiva em relação ao questionário e ao projeto.

Conclusões. A quantidade e o teor dos comentários espontaneamente enviados indicaram expressiva receptividade ao projeto e aprovação da estratégia de redução de danos pelo públicoalvo. O envolvimento dos participantes pôde ser observado em todos os comentários, sugerindo que a continuidade do projeto é urgente e oportuna.

Palavras-chave N-metil-3,4-metilenodioxianfetamina, psicotrópicos, redução do dano, Brasil.

Ainda que não tenham sido realizadas pesquisas epidemiológicas longitudinais relativas ao ecstasy no Brasil, o

\footnotetext{
1 Universidade de São Paulo (USP), Instituto de Psicologia, Departamento de Psicologia Experimental. Enviar correspondência para Stella Pereira Almeida no seguinte endereço: Universidade de São Paulo, Instituto de Psicologia, PSE, Avenida Prof. Mello Moraes 1721, CEP 05508-900, São Paulo, SP, Brasil. Fone: +55-11-3091.4176; fax: +55-11-3091.4357; e-mail: stella@usp.br
}

seu consumo parece seguir a tendência de crescimento alarmante constatada em diversos países (1-5). São indicações objetivas de que o consumo de ecstasy está em expansão no Brasil o aumento no volume de apreensões e a maior visibilidade na mídia, as declarações de freqüentadores de casas noturnas sobre a facilidade de obtenção de comprimidos e os relatos de profis- sionais de saúde que atuam na área do uso de drogas sobre o crescente número de casos atendidos $(6,7)$.

Embora haja indícios de que o uso de ecstasy tenha conseqüências adversas e possa ser neurotóxico em seres humanos, persistem as controvérsias (8-10). No entanto, sabe-se que existe uma relação entre o uso de ecstasy e problemas de memória, quadros psi- 
copatológicos agudos e casos esporádicos de intoxicação levando a coma ou morte (11-18). Além disso, indivíduos sob o efeito do ecstasy apresentam um aumento na freqüência de comportamentos de risco tais como dirigir sob o efeito de drogas, usar concomitantemente diferentes psicoativos, fazer exercícios físicos em ambientes muito quentes, alimentar-se e dormir pouco e expor-se mais freqüentemente a práticas sexuais de risco (19-22). Portanto, o uso de ecstasy pode efetivamente comportar risco, não só para a saúde de seus consumidores, mas também para aqueles que são expostos ao comportamento dos indivíduos sob o efeito da droga. Dessa forma, justifica-se a realização de intervenções preventivas para minimizar os danos do uso dessa droga, iniciativa da qual não se tem notícia até o momento no Brasil.

As campanhas preventivas baseadas no amedrontamento, ou seja, que divulgam informações exacerbando os malefícios das drogas, e cujo objetivo é a erradicação de seu consumo, nem sempre são eficazes (23, 24). Assim, outras estratégias têm sido propostas. Uma abordagem alternativa é a redução de danos (RD) (25), que considera utópica a erradicação do consumo de drogas e parte do pressuposto de que existem indivíduos que escolhem usálas, mas considera possível reduzir os riscos do uso. Tal estratégia ainda suscita polêmica, particularmente pelo temor de que possa estimular o consumo, e também pela suspeita de que apenas reduzir ou minimizar os riscos não seja suficiente.

Em relação ao ecstasy, diversos pesquisadores verificaram que os usuários têm conhecimento de estar correndo riscos quando usam a droga, mas nem por isso desejam interromper o consumo (26-29). Uma pesquisa diagnóstica realizada com usuários brasileiros verificou que a "preocupação com a saúde física" é o fator que mais interfere na freqüência de uso da droga, mas que uma minoria de usuários deseja diminuir ou interromper o uso (26).

Levando em consideração esses aspectos, o projeto Baladaboa elegeu a
RD como estratégia de intervenção preventiva particularmente adequada para usuários de ecstasy. Vale observar que, no Brasil, a Política Nacional Antidrogas, elaborada pela Secretaria Nacional Antidrogas (SENAD), aprova a RD explicitamente em uma de suas diretrizes, a saber, "apoiar e divulgar as pesquisas científicas submetidas e aprovadas por comitê de ética, realizadas na área de redução de danos para o aprimoramento e a adequação da política e de suas estratégias" (30).

O projeto Baladaboa, desenvolvido no Laboratório de Psicofarmacologia do Instituto de Psicologia da Universidade de São Paulo (USP), prevê três etapas: 1) pesquisa diagnóstica on-line, 2) intervenção e 3) avaliação. A primeira etapa, já realizada, é fundamental, dado que qualquer intervenção preventiva é tanto mais efetiva quanto mais adequada for a seu público-alvo. O diagnóstico foi feito através de um questionário on-line anônimo dirigido a usuários dessa droga (26). No site em que se encontrava o questionário a ser respondido, http://www.psicofarma cousp.psc.br/, também eram disponibilizadas informações sobre o projeto e sobre a RD. Ao final do questionário on-line, foi oferecido e utilizado, por centenas de participantes, um espaço para comentários espontâneos.

O objetivo do presente trabalho foi analisar o conteúdo dos comentários espontâneos feitos pelos participantes na primeira etapa do projeto Baladaboa, ou seja, na pesquisa diagnóstica on-line. Pretende-se assim ampliar o conhecimento sobre o universo dos usuários de ecstasy no Brasil, fornecendo um importante subsídio para a efetivação de programas de prevenção ao uso dessa droga.

\section{MATERIAIS E MÉTODOS}

O projeto foi aprovado pela Comissão de Ética em Pesquisa Humana do Instituto de Psicologia da USP. A análise de conteúdo se baseou no material escrito por participantes no último campo do questionário on-line disponível no site do projeto Baladaboa. O campo trazia a seguinte ins- trução: "Se quiser fazer sugestões, críticas ou dar opiniões utilize este espaço". O questionário diagnóstico online foi desenvolvido especificamente para o estudo realizado e será objeto de outro artigo. Em resumo, era direcionado aos usuários de ecstasy, exigia em torno de 20 minutos para ser respondido e era composto por 39 questões sobre padrões de uso de ecstasy e outras drogas, dados sociodemográficos e informações básicas sobre a droga, como efeitos e conseqüências do uso. $\mathrm{O}$ recrutamento dos participantes foi realizado através de e-mail, banners e artigos em sites, jornais e revistas. O questionário esteve disponível entre agosto de 2004 e fevereiro de 2005.

\section{Participantes e procedimentos de análise}

Dentre os 1140 indivíduos que responderam ao questionário diagnóstico on-line, 412 utilizaram o campo ao final do questionário para emitir algum comentário. Estes compõem a amostra não-probabilística por conveniência do presente estudo.

Em primeiro lugar, foi realizada uma comparação entre os participantes que fizeram comentários e os que não os fizeram quanto a dados sociodemográficos e dados relativos ao uso de ecstasy, através dos testes $t$ e qui-quadrado $\left(\chi^{2}\right)$. Em seguida, os comentários foram submetidos à análise de conteúdo, uma técnica utilizada para estudar unidades de comunicação. A técnica consiste na criação de categorias para classificar essas unidades, que podem ser palavras, temas ou tópicos. A comunicação é fracionada de acordo com regras previamente estipuladas (31).

Com base nessa técnica, e seguindo um procedimento anteriormente utilizado pelas autoras (32), foi feita uma análise do conteúdo dos discursos presentes nos 412 comentários digitados pelos participantes, de acordo com as seguintes regras: 1) foi identificada a idéia central do comentário, caracterizada como o conteúdo essencial do discurso apreendido (33); 2) os comentários que expressavam mais de uma idéia foram fracionados em segmentos; 
3) o tópico principal de cada segmento foi explicitado em uma expressãochave, correspondente à transcrição literal de parte do discurso "que permite o resgate do essencial do conteúdo discursivo dos segmentos em que se divide o depoimento" (33, p. 18); 4) tendo por base a sua equivalência, as expressões-chave registradas foram agrupadas em cinco categorias de discurso: irrelevante, drogas, depoimentos, questionário e projeto; 5) cada segmento foi então classificado em uma dessas cinco categorias; 6) quando pertinente, os segmentos foram ainda classificados em subcategorias.

A categoria denominada "irrelevante" não foi subdividida e incluiu saudações, além de comentários irreverentes e manifestações ideológicas. A categoria "drogas" foi subdividida nas subcategorias "sobre o esctasy", "sobre outras drogas" (considerações sobre causas, efeitos e conseqüências do uso de ecstasy ou de outras drogas) e "informações sobre o ecstasy" (segmentos que solicitavam esclarecimentos sobre a droga ou que apontavam a informação como uma questão fundamental relacionada a seu uso).

A categoria "depoimentos" foi subdividida nas subcategorias "sexualidade", "vida pessoal", "drogas" e "ecstasy", que incluíram comentários acerca de vivências pessoais. A categoria "questionário" foi subdividida nas subcategorias "questionário negativo", "questionário positivo", "questionário neutro" e "resultados", que incluíram comentários sobre o questionário propriamente dito, a formulação das questões, o uso de certos critérios e a solicitação de acesso aos resultados da pesquisa. A categoria "projeto" também não foi subdividida e incluiu os comentários relativos ao projeto Baladaboa.

Três juízes avaliaram independentemente a segmentação dos comentários, a atribuição de expressões-chave aos segmentos e a sua classificação em categorias e subcategorias. Em caso de divergência, considerou-se válida a classificação apontada por dois dos três juízes. Os três juízes, pós-graduados em psicologia, tinham experiência prévia em análise de conteúdo.

\section{RESULTADOS}

Os 412 participantes que fizeram comentários correspondem a 36,1\% da amostra de respondentes do questionário diagnóstico on-line ( $\mathrm{n}=1$ 140). Não houve diferenças entre o grupo de participantes que fez comentários e o grupo que não os fez. A tabela 1 mostra as características sociodemográficas e as características relativas ao uso de ecstasy (inclusive quanto à dependência) (34) dos participantes que fizeram comentários.

\section{Análise dos comentários}

Dentre os 412 comentários recebidos, 38 indivíduos enviaram endereços de e-mail e dois enviaram telefone de contato, solicitando resultados e colocando-se à disposição para fornecer maiores informações ou para participar de outras pesquisas. A todos os participantes que enviaram os seus endereços eletrônicos foi remetido um e-mail agradecendo a participação e informando que o tra- balho resultante da pesquisa diagnóstica realizada seria disponibilizado na Internet.

Foram identificados 915 segmentos nos 412 comentários. O número máximo de segmentos por comentário foi 9, e a média foi de 2,25 segmentos por comentário. Do conjunto desses 915 segmentos, $9,8 \%$ foram classificados na categoria "irrelevante", 16,4\% na categoria "drogas", 18,1\% na categoria "depoimentos", 25,1\% na categoria "questionário" e 30,6\% na categoria "projeto". A tabela 2 mostra exemplos de comentários para cada categoria.

Os relatos de experiências com o uso de ecstasy foram os mais freqüentes dentre os depoimentos, correspondendo a mais de metade deles, sendo também relatadas experiências com outras drogas. Nas subcategorias "sexualidade" e "vida pessoal" os participantes mostraram-se principalmente interessados em esclarecer as respostas para evitar interpretações errôneas. Na categoria "questionário", a subcategoria com mais segmentos foi "questionário negativo", sendo a falta de opções em algumas perguntas a crítica

TABELA 1. Características sociodemográficas e relativas ao uso de ecstasy dos participantes $^{\mathrm{a}}$ de pesquisa on-line, Brasil, 2005

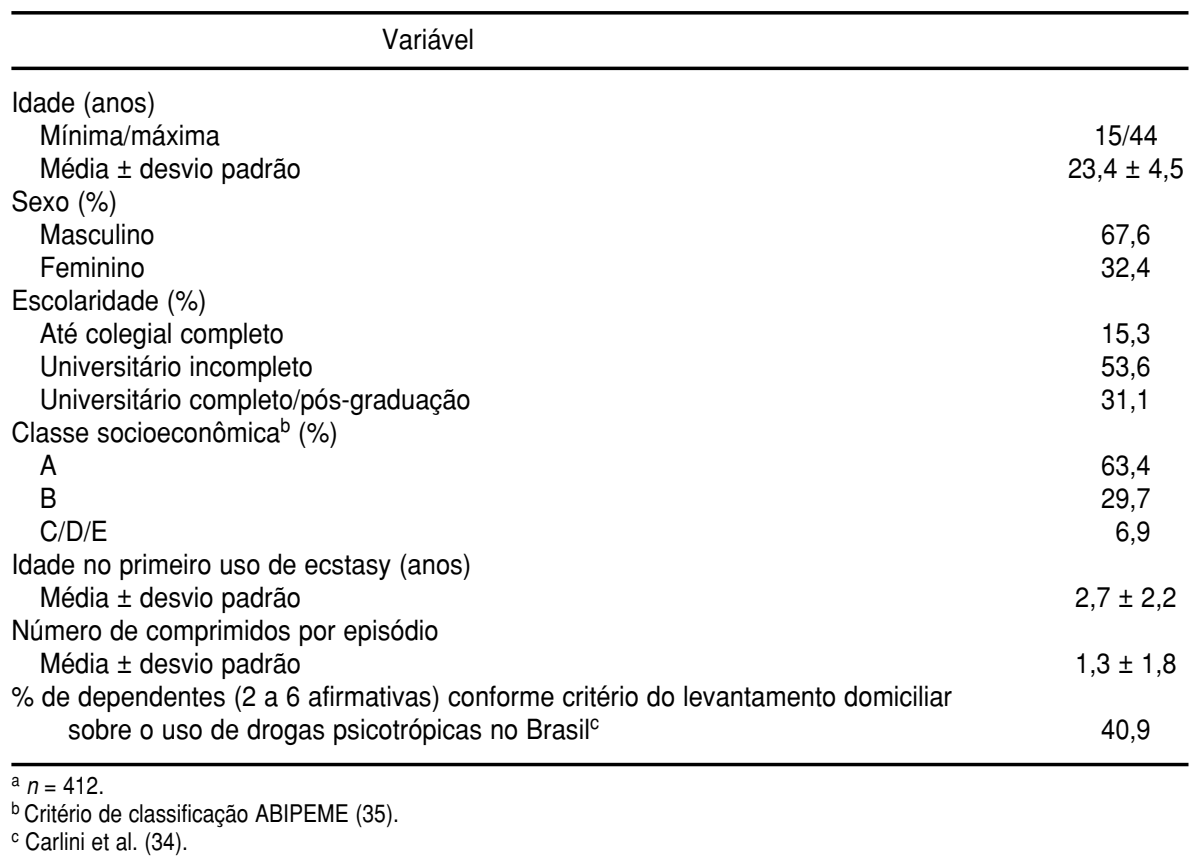


TABELA 2. Comentários espontâneos dos participantes da pesquisa on-line sobre uso de ecstasy, Brasil, 2005

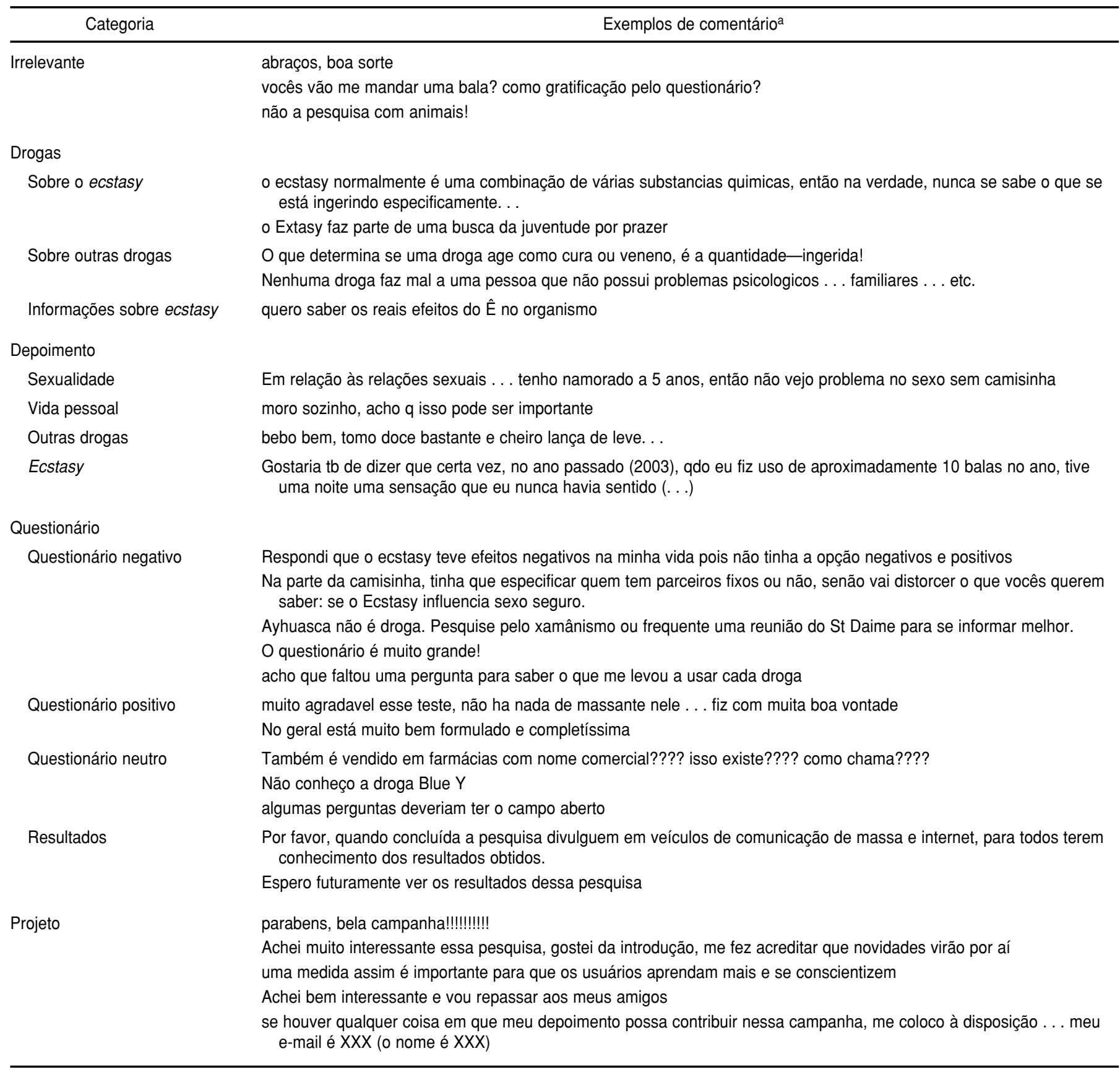

a Os comentários foram transcritos ipsis litteris da pesquisa, sem correção ortográfica ou qualquer modificação ao texto. Apenas os e-mails e telefones foram omitidos.

mais freqüente. Também foram feitas críticas em relação à classificação socioeconômica utilizada (critério ABA/ABIPEME de 1982) (35), à pergunta sobre sexo seguro e à inclusão de ayahuasca na lista de drogas. Muitas vezes, as críticas foram seguidas de esclarecimentos em relação às respostas dadas. Ainda em relação a essa subcategoria, houve tanto reclamações quanto à extensão do questionário como à falta de perguntas julgadas importantes pelos participantes. Em "questionário neutro", os segmentos, na maior parte das vezes, solicitavam informações relacionadas às perguntas, particularmente esclarecimentos relativos a uma droga fictícia que constava do questionário.

A categoria projeto foi a que conteve mais segmentos. Tipicamente, os seg- 
mentos dessa categoria foram elogios à iniciativa, estímulos à execução do projeto, discursos sobre a importância do projeto ou sobre a RD para o uso de ecstasy e comentários nos quais os participantes se ofereciam para dar maiores esclarecimentos ou participar de outras pesquisas semelhantes.

\section{DISCUSSÃO}

Em primeiro lugar, vale dizer que a quantidade de comentários espontaneamente enviados foi expressiva, particularmente tendo em vista que o questionário on-line demandava em torno de 20 minutos para ser respondido. Em se tratando do uso de uma droga ilegal, surpreende que quase $10 \%$ dos participantes que fizeram comentários tenham enviado contatos eletrônicos ou telefônicos, demonstrando o interesse em participar mais ativamente do projeto e a confiança quanto à seriedade da proposta. É importante observar que a participação dos usuários de drogas nos programas de prevenção a eles direcionados é absolutamente condizente com a abordagem de RD, que prevê que os usuários sejam, sempre que possível, agentes multiplicadores (36-38). Portanto, o envio de tais contatos é um excelente prognóstico da participação voluntária de usuários de ecstasy nas etapas de intervenção e avaliação previstas no projeto Baladaboa.

O caráter espontâneo dos comentários é algo que os valoriza sobremaneira. É de se supor que tais comentários expressem questões centrais para pessoas especialmente envolvidas com o tema, sendo uma fonte de informação valiosa, diversificada e obtida com um mínimo de estimulação. Vale ressaltar que a metodologia qualitativa, como pode ser classificada a análise de discurso utilizada neste estudo, auxilia no trabalho de construção do objeto estudado, facilitando a descoberta de dimensões ainda não conhecidas. É uma abordagem particularmente adequada a áreas para as quais não há um conhecimento teórico e conceitual plena- mente desenvolvido, sendo ideal para o estudo de populações de difícil acesso e de epidemiologia não demarcada, como os usuários de ecstasy no Brasil $(39,40)$.

De forma geral, o envolvimento dos participantes com o projeto e com a pesquisa pôde ser observado em todos os comentários. Mesmo os segmentos da categoria "irrelevante" mostraram, freqüentemente, uma disposição afável para com os pesquisadores. Até quando continham irreverências, essas observações indicavam empatia ou humor, sem traços de agressividade.

Na categoria "depoimentos", observou-se freqüentemente o desejo de colaborar com os pesquisadores, na tentativa de evitar interpretações errôneas. Nessa categoria também foram relatadas com freqüência experiências pessoais com drogas, sugerindo que um espaço destinado ao compartilhamento de tais experiências, com a mediação de um especialista na área, seria apreciado por usuários.

$\mathrm{Na}$ categoria "drogas", ficou evidente, principalmente, a intenção de aprofundar questões ligadas ao assunto, fossem ou não específicas do ecstasy. Nessa categoria observaram-se opiniões de cunho ideológico, análises sobre possíveis causas e soluções para a questão das drogas, revelando indivíduos que ponderam a situação do usuário e da política relativa às drogas. Em alguns comentários dessa categoria, foi abordado exclusivamente o tema prazer, como naqueles que faziam a apologia do uso de drogas e exaltavam os seus efeitos. Porém, a grande maioria dos comentários era abrangente e fazia análises elaboradas, o que sugere que esses comentários tenham sido feitos por pessoas para as quais o uso de substâncias ocupa um espaço mais amplo do que o desfrute fugaz de um efeito prazeroso. Em relação aos comentários sobre o "questionário", também pôde ser observado que, de forma geral, houve intenção de colaborar, mesmo entre aqueles que criticaram o formato ou o conteúdo das questões. A solicitação dos resultados da pesquisa, subcategoria presente em quase um quinto dos comentários, mostrou o interesse dos usuários em conhecer o seu próprio universo. Em atenção a essa demanda, em dezembro de 2005 a tese advinda de tal pesquisa foi disponibilizada na íntegra no site www.teses.usp.br e também no site do projeto.

Em relação à categoria "projeto", a que mais comentários suscitou, é importante notar que foram enviadas observações extremamente favoráveis à proposta do projeto Baladaboa. O fato de metade dos comentários tecer elogios à iniciativa, incentivar os pesquisadores a executar as ações propostas, ou oferecer ajuda no que fosse necessário é indício de que um projeto de RD, caso implantado, terá grande aceitação entre os usuários de ecstasy.

Somada à ascensão do consumo da droga, ao seu comprovado potencial tóxico e à inexistência de programas preventivos direcionados a usuários brasileiros até o momento, a receptividade do público-alvo ao projeto Baladaboa, constatada na presente análise, indica que sua efetiva implantação não é apenas necessária, mas urgente e apropriada ao momento. Quanto antes for implantado o primeiro programa preventivo para o uso de ecstasy, maior será o número de usuários experimentais e recentes atingidos, aumentando as chances de que incorporem atitudes de RD.

Em suma, esperamos que o presente trabalho contribua para sensibilizar as autoridades responsáveis pela política pública brasileira em relação a drogas para a importância da realização de intervenções que atendam à demanda espontaneamente manifestada pelos usuários.

Agradecimentos. Stella Pereira Almeida recebeu bolsa de doutorado da Coordenação de Aperfeiçoamento de Pessoal de Nível Superior (CAPES) e bolsa de pós-doutorado da Fundação de Amparo à Pesquisa do Estado de São Paulo (FAPESP). Maria Teresa Araújo Silva recebeu auxílio do Conselho Nacional de Desenvolvimento Científico e Tecnológico (CNPq). 


\section{REFERÊNCIAS}

1. Degenhardt L, Copeland J, Dillon P. Recent trends in the use of "club drugs": an Australian review. Subst Use Misuse. 2005;40 (9-10):1241-56.

2. McCambridge J, Mitcheson L, Winstock A, Hunt N. Five-year trends in patterns of drug use among people who use stimulants in dance contexts in the United Kingdom. Addiction. 2005;100(8):1140-9.

3. Strote J, Lee JE, Wechsler H. Increasing MDMA use among college students: results of a national survey. J Adolesc Health. 2002;30 (1):64-72.

4. Wilkins C, Bhatta K, Pledger M, Casswell S. Ecstasy use in New Zealand: findings from the 1998 and 2001 National Drug Surveys. N Z Med J. 2003;116(1171):U383.

5. Ahmad K. Increased use of amphetaminetype stimulants threatens east Asian countries. Lancet. 2002;359(9321):1927.

6. de Almeida SP, Silva MT. Histórico, efeitos e mecanismo de ação do êxtase (3-4 metilenodioximetanfetamina): revisão da literatura. Rev Panam Salud Publica. 2000;8(6):393-402.

7. United Nations Office on Drugs and Crime (UNODC). World Drug Report. New York: UNODC; 2006. Disponível em: http://www. unodc.org/unodc/world_drug_report.html. Acessado em novembro de 2007.

8. Dumont GJ, Verkes RJ. A review of acute effects of 3,4-methylenedioxymethamphetamine in healthy volunteers. J Psychopharmacol. 2006;20(2):176-87.

9. de Win MM, Reneman L, Jager G, Vlieger EJ, Olabarriaga SD, Lavini C, et al. A prospective cohort study on sustained effects of low-dose ecstasy use on the brain in new ecstasy users. Neuropsychopharmacology. 2007;32(2):458-70.

10. Jager $\mathrm{G}$, de Win MM, Vervaeke HK, Schilt $\mathrm{T}$, Kahn RS, van den Brink W, et al. Incidental use of ecstasy: no evidence for harmful effects on cognitive brain function in a prospective fMRI study. Psychopharmacology (Berl). 2007;193(3):403-14.

11. Wareing M, Fisk JE, Murphy PN. Working memory deficits in current and previous users of MDMA ('ecstasy'). Br J Psychol. 2000;91 (Pt 2):181-8.

12. Parrott AC. Human research on MDMA (3,4methylene-dioxymethamphetamine) neurotoxicity: cognitive and behavioural indices of change. Neuropsychobiology. 2000;42(1):17-24.

13. Morgan MJ, McFie L, Fleetwood H, Robinson JA. Ecstasy (MDMA): are the psychological problems associated with its use reversed by prolonged abstinence? Psychopharmacology (Berl). 2002;159(3):294-303.

14. McGuire PK, Cope H, Fahy TA. Diversity of psychopathology associated with use of 3,4methylenedioxymethamphetamine ('Ecstasy'). Br J Psychiatry. 1994;165(3):391-5.

15. MacInnes N, Handley SL, Harding GF. Former chronic methylenedioxymethampheta- mine (MDMA or ecstasy) users report mild depressive symptoms. J Psychopharmacol. 2001;15(3):181-6.

16. Gamma A, Frei E, Lehmann D, PascualMarqui RD, Hell D, Vollenweider FX. Mood state and brain electric activity in ecstasy users. Neuroreport. 2000;11(1):157-62.

17. Thomasius $R$, Petersen $K$, Buchert $R$, Andresen B, Zapletalova P, Wartberg L, et al. Mood, cognition and serotonin transporter availability in current and former ecstasy (MDMA) users. Psychopharmacology (Berl). 2003;167(1):85-96.

18. Cohen RS. Subjective reports on the effects of the MDMA ('ecstasy') experience in humans. Prog Neuropsychopharmacol Biol Psychiatry. 1995;19(7):1137-45.

19. Almeida SP, Silva MTA. Ecstasy (MDMA): Effects and patterns of use reported by users in São Paulo. Rev Bras Psiquiatr. 2003;25(1): $11-17$.

20. Lee SJ, Galanter M, Dermatis H, McDowell D. Circuit parties and patterns of drug use in a subset of gay men. J Addict Dis. 2003;22(4): $47-60$.

21. Logan BK, Couper FJ. 3,4-Methylenedioxymethamphetamine (MDMA, ecstasy) and driving impairment. J Forensic Sci. 2001; 46(6):1426-33.

22. Green AR, Sanchez V, O'Shea E, Saadat KS, Elliott JM, Colado MI. Effect of ambient temperature and a prior neurotoxic dose of 3,4methylenedioxymethamphetamine (MDMA) on the hyperthermic response of rats to a single or repeated ('binge' ingestion) low dose of MDMA. Psychopharmacology (Berl). 2004; 173(3-4):264-9.

23. Carlini-Cotrim B. Drogas na escola: prevenção, tolerância e pluralidade. Em: Aquino JGO, ed. Drogas na escola: alternativas teóricas e práticas. São Paulo: Summus; 1998. Pp. 19-30.

24. Cuijpers P. Effective ingredients of schoolbased drug prevention programs. A systematic review. Addict Behav. 2002;27(6):1009-23.

25. Moreira FG, Silveira DX, Andreoli SB. Situações relacionadas ao uso indevido de drogas nas escolas públicas da cidade de São Paulo. Rev Saude Publica. 2006;40(5):810-7.

26. Almeida SP. Sobre o uso de ecstasy: uma pesquisa com vistas à formulação de intervenção preventiva [tese]. São Paulo: USP; 2005. Disponível em: http:/ / www.teses.usp.br/teses/ disponiveis/47/47135/tde-08022006-110827/. Acessado em novembro de 2007.

27. Allott K, Redman J. Patterns of use and harm reduction practices of ecstasy users in Australia. Drug Alcohol Depend. 2006;82(2): 168-76.

28. Murphy PN, Wareing M, Fisk J. Users' perceptions of the risks and effects of taking ecstasy (MDMA): a questionnaire study. J Psychopharmacol. 2006;20(3):447-55.
29. Levy KB, O'Grady KE, Wish ED, Arria AM. An in-depth qualitative examination of the ecstasy experience: results of a focus group with ecstasy-using college students. Subst Use Misuse. 2005;40(9-10):1427-41.

30. Brasil, Secretaria Nacional Antidrogas (SENAD). Política Nacional sobre drogas. In: Brasília; SENAD; 2005. Disponível em: http://www.senad.gov.br/. Acessado em novembro de 2007.

31. Aaker DA, Kumar V, Day GS. Marketing research. Nova Iorque: John Wiley \& Sons; 1988.

32. Barros RS, Almeida SP, Magalhães MP, Silva MTA. Idéias e imagens suscitadas em universitários numa pesquisa sobre drogas: uma contribuição ao trabalho preventivo. Bol Psicol. 1992;42(96/97):15-26.

33. Lefèvre F, Lefèvre AMC, Teixeira JJV. O discurso do sujeito coletivo: uma nova abordagem metodológica em pesquisa qualitativa. Caxias do Sul: EDUCS; 2000.

34. Carlini EA, Galduróz JCF, Noto AR, Nappo SA. Primeiro levantamento domiciliar sobre o uso de drogas psicotrópicas no Brasil. São Paulo: Secretaria Nacional Antidrogas; 2002. Disponível em: http://www.unifesp.br/ dpsicobio/cebrid/levantamento_brasil/parte 2.pdf. Acessado em novembro de 2007.

35. Almeida PM, Wickerhauser H. O Critério ABA/ABIPEME - em busca de uma atualização. Um estudo e uma proposta submetidos à $\mathrm{ABA}$ e à $\mathrm{ABIPEME}$. São Paulo: Documento de circulação restrita da $\mathrm{ABA}$ e da ABIPEME; 1991.

36. Carlson RG, Falck RS, McCaughan JA, Siegal HA. MDMA/Ecstasy use among young people in Ohio: perceived risk and barriers to intervention. J Psychoactive Drugs. 2004;36(2): 181-9.

37. Harm Reduction Coalition. Promoting the health and dignity of those impacted by drug use. Disponível em: http://www.harm reduction.org/. Acessado em 30 de maio de 2006.

38. Marlatt GA. Redução de danos: estratégias práticas para lidar com comportamentos de alto risco. Porto Alegre: Artmed; 1999.

39. Serapione M. Métodos qualitativos e quantitativos na pesquisa social em saúde: algumas estratégias para a integração. Cienc Saude Coletiva. 2000;5(1):187-192

40. Beck J, Rosenbaum M. Pursuit of Ecstasy. The MDMA experience. Albany: State University of New York; 1994.

Manuscrito recebido em 19 de junho de 2006. Aceito em versão revisada el 30 de julho de 2007. 
ABSTRACT Objective. To analyze the contents of spontaneous comments posted by ecstasy users participating in an online survey that constitutes phase one of a harm reduction project (projeto Baladaboa) aimed at users of this drug.

Analysis of spontaneous comments posted by ecstasy users participating in an online survey

Key words N-methyl-3,4-methylenedioxyamphetamine, psychotropic drugs, harm reduction, Brazil.
Method. Of the 1140 individual respondents, 412 posted unprompted comments. These comments were grouped into five categories (irrelevant, drugs, testimonies, survey, and project) and submitted to content analysis. Salutations, irreverent comments, and ideological remarks were considered "Irrelevant." Comments on the causes, effects, and consequences of using ecstasy or other drugs; requests for information on the drug; or comments indicating that information is a crucial issue associated with the use of ecstasy were labeled "Drugs." Personal stories made up the group called "Testimonies." Any comments concerning the questionnaire itself, the formulation of questions, the use of certain criteria, and requests to see survey results were designated "Survey." Comments specifically about the Baladaboa project were categorized as "Project."

Results. Content analysis revealed 915 segments among the 412 comments. The percent distribution of segments among the groups were: irrelevant, 9.8\%; drugs, $16.4 \%$; testimonies, $18.1 \%$; survey, $25.1 \%$; and project, $30.6 \%$. Most of the comments concerning the survey and the project were positive.

Conclusions. The number and content of the spontaneous comments indicate that the project, as well as the harm reduction strategy, were being well received by the target audience. The participants expressed their engagement with the project and suggested that its continuation is both timely and urgent.

Key words

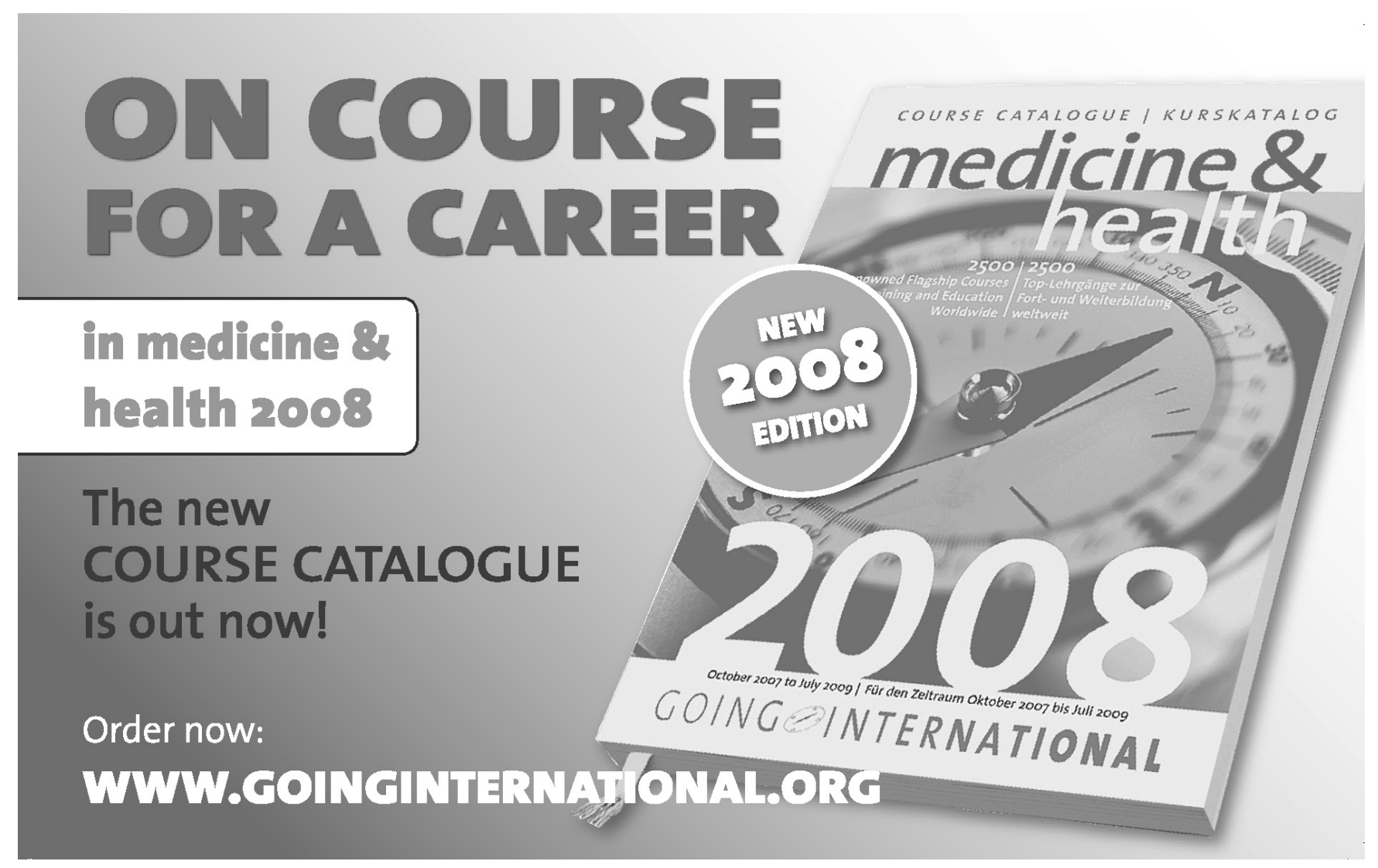

